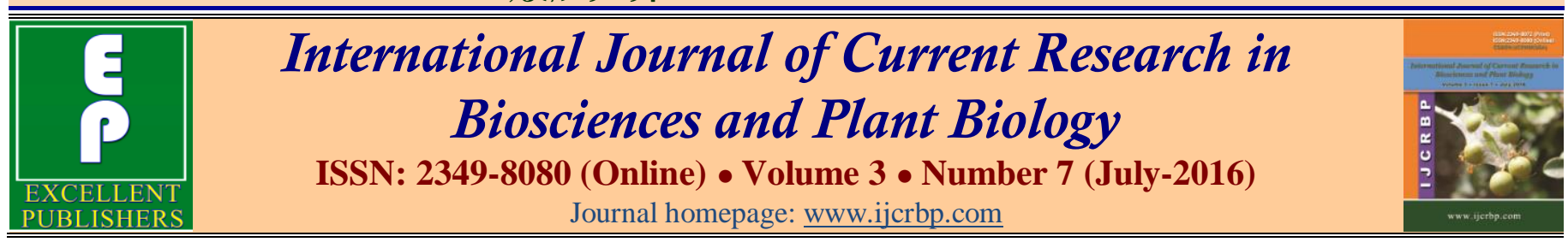

Original Research Article

doi: http://dx.doi.org/10.20546/ijcrbp.2016.307.013

\title{
Molecular Cloning and Sequence Analysis of GbWRKY31, A Novel Transcription Factor Gene from Ginkgo biloba
}

\author{
Xiaoqiang Li, Jiabao Ye, Xiaohui Wang, Weiwei Zhang and Feng Xu* \\ College of Horticulture and Gardening, Yangtze University, Jingzhou 434025, China \\ *Corresponding author.
}

\begin{abstract}
WRKY proteins are a class of plant-specific transcription factors involved in stress response signaling pathways. In this work, a novel WRKY transcription factor gene, named after GbWRKY31, was isolated by RT-PCR method from the Ginkgo biloba. The full-length cDNA of GbWRKY31 was $1738 \mathrm{bp}$ and contained a $1281 \mathrm{bp}$ open reading frame (ORF) encoding 427 amino acids. The estimated isoelectric point (pI) and molecular weight of the putative GbWRKY3I protein were 9.10 and $47.05 \mathrm{kDa}$, respectively. Homology analysis indicated that the deduced GbWRKY31 protein was highly homologous to other WRKY Proteins from different species and had a typical WRKY conserved domain database. Phylogenetic analysis showed that GbWRKY3I belongs to the WRKY IIb group. The results suggest that GbWRKY31 belongs to WRKY families and may be involved in the modulation of senescence and defense in G. biloba.
\end{abstract}

Article Info

\section{Introduction}

Ginkgo biloba, the oldest species in the world, has been growing for nearly 200 million years on the earth (He et al., 2009). It has many good characters, such as diseaseresistance, drought-resistance (Cheng et al., 2013). Recent studies have shown that WRKY transcription factors were play an important role in plant growth involved in physiological metabolism process of biological signal stimulation and defense responses against pathogens.

It has been reported that WRKY transcription factors are crucial regulators in the transcription and disease resistance (Eulgem and Somssich, 2007). For example, AtWRKY70 in Arabidopsis thaliana, appears to affect the balance between signaling branches promoting SA-dependent and suppressing JA-dependent responses ( $\mathrm{Li}$ et al., 2006); The rice carrying
OsWRKY71 gene had a stronger resistance to Xanthomonas oryzae, in addition, the expression of disease resistance related gene was also significantly increased (Liu et al., 2007).

The WRKY protein includes one or more WRKY domains, which were composed of conserved WRKYGQK peptide and typical zinc finger motif, $\mathrm{CX}_{4-5} \mathrm{CX}_{22-23} \mathrm{HXH}\left(\mathrm{C}_{2} \mathrm{H}_{2}\right)$ or $\mathrm{CX}_{7} \mathrm{CX}_{23} \mathrm{HXC}\left(\mathrm{C}_{2} \mathrm{HC}\right)$, at the $\mathrm{N}$-terminal and the $\mathrm{C}$-terminal separately (Eulgem et al., 2000). According to the quantity of WRKY domain and the zinc finger motif, WRKY gene families can be divided into three groups: Group I included two WRKY domains with a $\mathrm{C}_{2} \mathrm{H}_{2}$ zinc-finger structure; Group II and III consist of only one WRKY domain with a $\mathrm{C}_{2} \mathrm{H}_{2}$ and a $\mathrm{C}_{2} \mathrm{HC}$ zinc-finger structure, respectively. Meanwhile, the group IIWRKY genes were further divided into IIa, IIb, IIc, IId, and IIe based on their primary amino acid sequence (Rushton et al., 2012). 
In terms of molecular biology, WRKY proteins perform themselves function through specifically combining with the W-box (5'-TTGACC/T-3'), which is the cis-element and the minimal consensus required for specific DNA binding (Rushton et al., 1996; Ciolkowski et al., 2008). W-box sequences generally exist in promoter region involving in stress response. Moreover, WRKY genes promoter regions also often have W-box (Maleck et al., 2000). In recent years, a lot of WRKY transcription factors had been identified, and then discovered the association with secondary metabolism. The function of AtWRKY75 has been confirmed in regulating $\mathrm{Pi}$ starvation responses (Devaiah et al., 2007). TcWRKY1 participates in regulation of taxol biosynthesis in Taxus chinensis cells (Li et al., 2013). Previously, there are few WRKY genes that had been isolated and characterized from $G$. biloba. At present, transcriptome analysis of $G$. biloba was performed by our laboratory research group designed through using Illumina HiSeq $^{\mathrm{TM}} 2500$ sequencing platform. Based on the transcriptome data, we cloned and characterized GbWRKY31 from G. biloba.

\section{Materials and methods}

\section{Plant material and reagents}

The leaves of ginkgo were collected from Botanical Garden of Yangtze University, China, and stored at -80 ${ }^{\circ} \mathrm{C}$ refrigerator immediately. Both the primers synthesis and DNA sequencing were performed by Shanghai Sangon Biotechnology Company, in China. Agarose Gel DNA purification Kit Ver.4.0, pMD19-T vector kit, AMV Reverse Transcriptase, RNase, PrimeScript ${ }^{\mathrm{TM}} 1$ st Strand cDNA Synthesis kit PrimeScript ${ }^{\mathrm{TM}}$ RT-PCR kit and Taq DNA polymerase were purchased from Takara Company, Dalian, China.

\section{RNA extraction and reverse transcription}

Total RNA was isolated from plant frozen plant tissues using the MiniBEST Plant RNA Extraction kit (TaKaRa, Dalian, China). According to the instructions of PrimeScript $^{\mathrm{TM}}$ 1st Strand cDNA Synthesis Kit (TaKaRa, Dalian, China), first-strand cDNA was synthesized.

\section{Cloning of GbWRKY31}

The specific primer GbWRKY31-F (5'-AAGGTTTCC AATGCGGATAAG-3') and GbWRKY31-R (5'-AGTG CCGCAGTGAAGTTGGGA-3') were designed using the software of DNAMAN6. The PCR was performed using the one-step RT-PCR kit (TaKaRa, Dalian, China) under the following conditions: per-denaturation at $94^{\circ} \mathrm{C}$ for 3 min; denaturation at $94^{\circ} \mathrm{C}$ for $30 \mathrm{~s}$, anneal at $58^{\circ} \mathrm{C}$ for $30 \mathrm{~s}$, and extension at $72^{\circ} \mathrm{C}$ for 40 s, by 35 cycles; extend at $72^{\circ} \mathrm{C}$ for $10 \mathrm{~min}$. The PCR product was purified and cloned into pMD19-T vector kit (TaKaRa, Dalian, China), followed by sequencing for confirmation.

\section{Bioinformatic analysis}

The sequence was analyzed by the bioinformatics software on websites (http://www.xpasy.org and http://www.ncbi.nlm.nih.gov), protein sequences were retrieved from NCBI GenBank. Sequence alignment was performed using ClustalX 2.0 and phylogenetic tree was constructed by neighbor-joining method using MEGA 6.0. The bootstrap statistical analysis was carried out with 1000 replicates.

\section{Results}

\section{Cloning and sequence analysis of $G b W R K Y 31$}

The cDNA sequence of GbWRKY31 gene was obtained according to a pair of specific primers and total RNA reverse transcription product. The length of GbWRKY3I was $1738 \mathrm{bp}$, and the gene contained 1281 bp-length open reading frame and encoding 427 amino acids (Fig. 1). We compared the nucleotide sequence of GbWRKY31 with the nucleotide sequences of other plants acquired from the NCBI database and found the nucleotide sequence of GbWRKY31 had high similarity with WRKY genes of other plants (Table 1).

\section{Characterization of the deduced GbWRKY31 protein}

The GbWRKY31 protein encodes 552 amino acids (Fig. 1). Using the online website (http://web.expasy.org/ compute_pi/), the molecular weight could be known and theoretical isoelectric point of the GbWRKY31 protein were $47.05 \mathrm{kDa}$ and 9.10, respectively. Using BLAST search of GeneBank and Vector NTI, the Fig. 2 shows that the similarity of the GbWRKY31 protein with other WRKY proteins was made. Sequencing analysis indicated that the predicted $G b W R K Y 31$ protein contains WRKY-motif and Zinc-finger motif. GbWRKY31 protein was $43-44 \%$ similarity to Group IIb WRKY proteins including EgWRKY31 of Eucalyptus grandis, PeWRKY31 of Populus euphratica, PkWRKY of Picrorhiza kurrooa, MaWRKY31 of Musa acuminata, CsWRKY31 of Cucumis sativus, VrWRKY31 of Vigna radiata, GmWRKY23 of Glycine max and GsWRKY6 of Glycine soja. 
Table 1. Nucleotide sequence of GbWRKY31 similarity to the WRKY genes of other plant species.

\begin{tabular}{llll}
\hline Species & GenBank no. & Identity & E-value \\
\hline Ziziphus jujuba & XM_016039435 & $97 \%$ & 5e-04 \\
Gossypium hirsutum & KF669851 & $93 \%$ & $1 \mathrm{e}-05$ \\
Gossypium hirsutum & KF031106.1 & $93 \%$ & $1 \mathrm{e}-05$ \\
Eucalyptus grandis & XM_010030989.1 & $88 \%$ & 5e-04 \\
Brassica rapa & NM_001301983.1 & $81 \%$ & $1 \mathrm{e}-04$ \\
Tarenaya hassleriana & XM_010531148.1 & $76 \%$ & $2 \mathrm{e}-12$ \\
Arabidopsis lyrata & XM_002872646.1 & $76 \%$ & $9 \mathrm{e}-12$ \\
Solanum tuberosum & KF280263.1 & $74 \%$ & $1 \mathrm{e}-15$ \\
\hline
\end{tabular}

Note: The nucleotide sequence of GbWRKY31 was 97\%, 93\%, 93\%, 88\%, 81\% similarity to ZjWRKY72 in Ziziphus jujuba, GhWRKY90 in Gossypium hirsutum, GhWRKY80 in Gossypium hirsutum, EgWRKY72 in Eucalyptus grandis, BrWRKY47 in Brassica rapa, respectively.

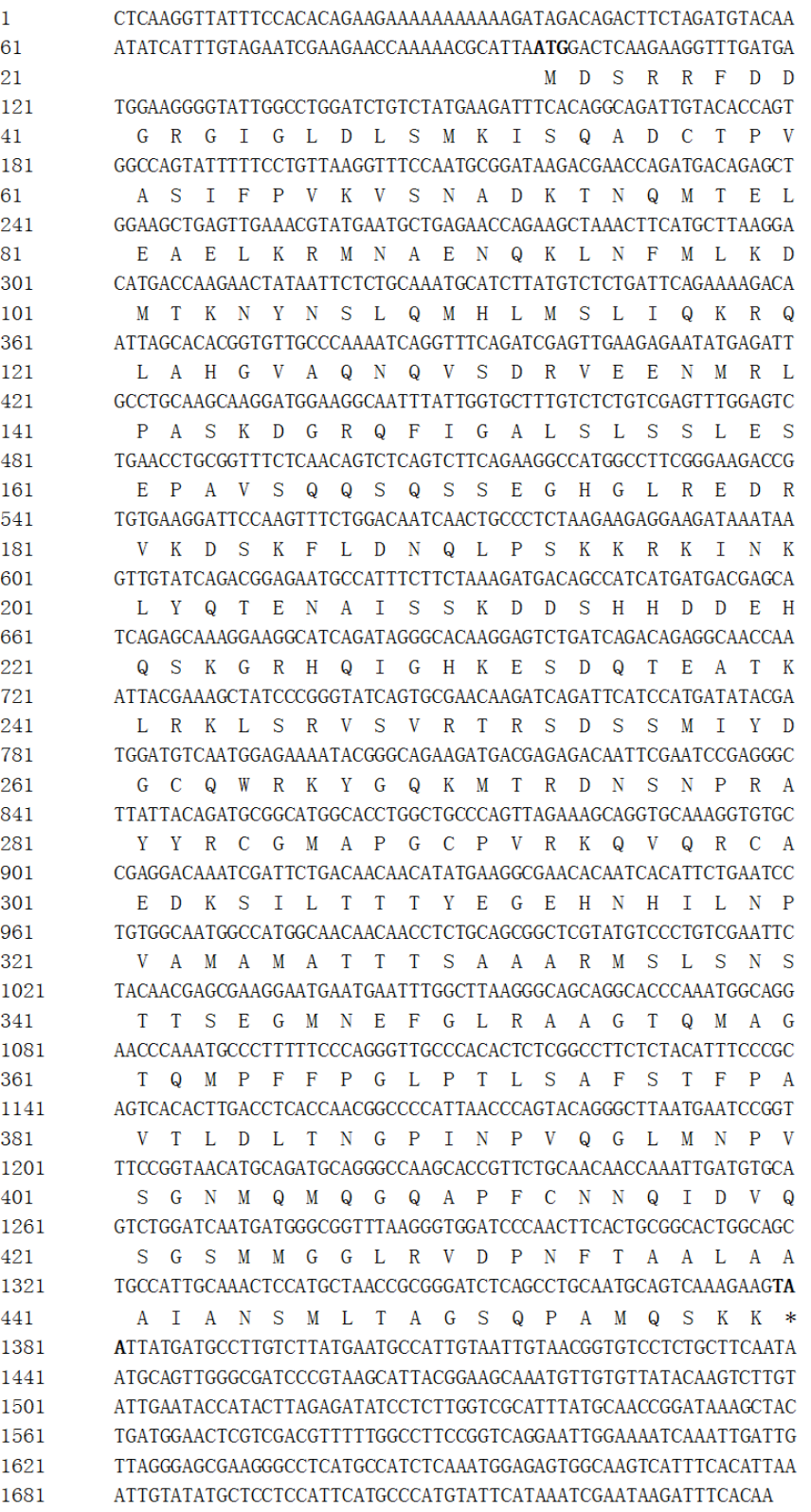

Fig. 1: Nucleotide sequence and deduced amino acid sequence of GbWRKY31.

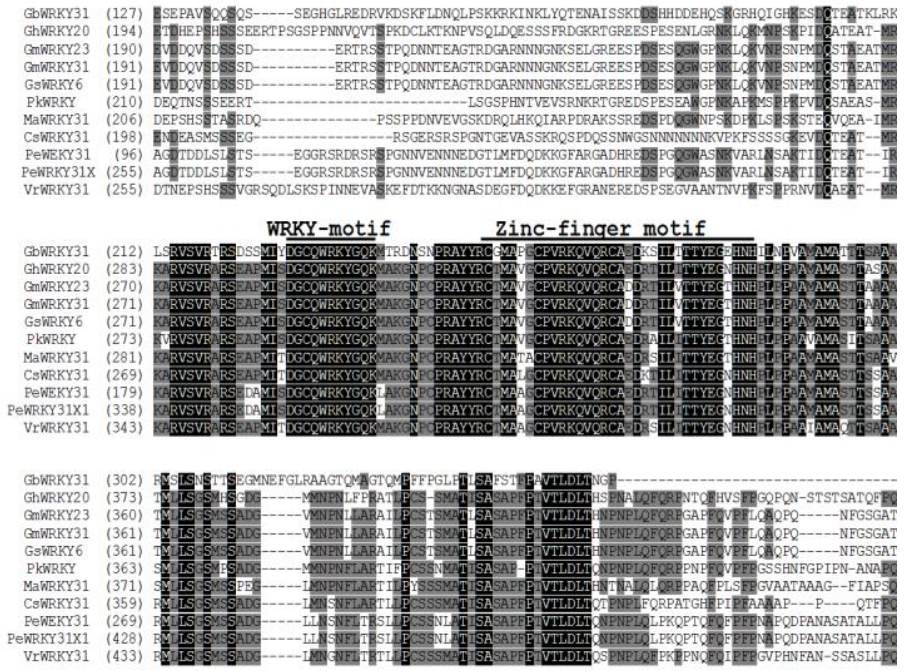

Fig. 2: Sequence multi-alignment of the deduced GbWRKY31 protein with other WRKY proteins. The accession numbers of WRKY proteins and translation of their names are shown as follows, GbWRKY31: Ginkgo biloba; GhWRKY20: Gossypium hirsutum AJT43303.1; GmWRKY23: Glycine max ABS18425.1; GmWRKY31: Glycin emax ABS18431.1; GsWRKY6: Glycine soja KHN40870.1; PkWRKY: Picrorhiza kurrooa ACI90292.1; MaWRKY31: Musa acuminata XP_009388735.1; CsWRKY31: Cucumis sativus XP_011658698.1; PeWRKY31: Populus euphratica XP_011033773.1; PeWRKY31x1: Populus euphratica XP_011047237.1; VrWRKY31: Vigna radiate XP_014498648.1. The completely identical amino acids are indicated with white foreground and black background. Shaded in gray are conservative sequences. Conserved motifs WRKY-motif and Zinc-finger motif are marked.

\section{Phylogenetic tree analysis of GbWRKY31}

The phylogenetic tree showing the evolutionary relationships among GbWRKY31 and other WRKY proteins was constructed with 17 WRKY proteins (Fig. $3)$. The evolutionary tree was divided into seven distinct categories. It is clearly to know kinship of the 17 species 
from (Fig. 3). The result indicated that GbWRKY31 was closely related to AtWRKY6 of Arabidopsis thaliana and TwWRKY of Taxus wallichiana. From the above, GbWRKY31 belongs to the group IIb of the WRKY families.

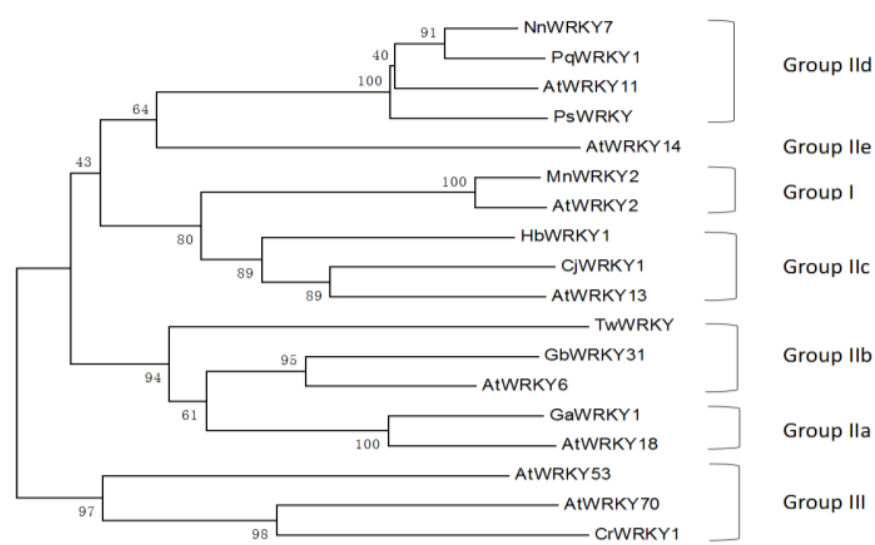

Fig. 3: Phylogenetic tree of the sequences of GbWRKY31 and other plants WRKY protein. Bootstrap value are expressed in percentages and placed at the nodes in the tree. The GenBank accession numbers of the WRKY proteins and translation of their names are shown, GbWRKY31: Ginkgo biloba; CjWRKY1: Coptis japonica BAF41990.1; AtWRKY13: Arabidopsis thaliana AEE87071.1; HbWRKY1: Hevea brasiliensis ADF45433.1; MnWRKY2: Morus notabilis XP_010092241.1; AtWRKY2: Arabidopsis thaliana AED96743.1; TwWRKY: Taxus wallichiana AEW91476.1; AtWRKY6: Arabidopsis thaliana AEE33948.1; GaWRKY1: Gossypium arboreum AAR98818.1; AtWRKY18: Arabidopsis thaliana AEE85961.1; AtWRKY14: Arabidopsis thaliana AEE31256.1; PsWRKY: Picea sitchensis ADE77495.1; AtWRKY11: Arabidopsis thaliana AEE85928.1; NnWRKY7: Nelumbo nucifera XP_010270802.1; PqWRKY1: Panax quinquefolius AEQ29014.1; AtWRKY53: Arabidopsis thaliana AEE84809.1; AtWRKY70: Arabidopsis thaliana AEE79517.1; CrWRKY1: Catharanthus roseus ADT82685.1.

\section{Discussion}

The GbWRKY31 gene was isolated from ginkgo in this study. The multiple sequence alignment by using bioinformatics analysis software indicated that GbWRKY31 had high identity with other WRKY genes cloned from other plants. The homologous sequence of WRKY gene among different plants implied that the WRKY gene belongs to the group IIb of the WRKY families and keep a strong conservation during the molecular evolution. The conserved domain motif function further indicated GbWRKY31 might involve in immune response in plants and might be the key of disease-resistant defense reaction (Peng et al., 2008; Ramiro et al., 2010).
WRKY transcription factors are involved in biological stress and non-biological stress, growth processes in plant. The functional diversity within WRKY genes determined the plant defense responses and regulation of vegetative growth. There was a research results show that AtWRKY6, also belonging to the WRKY IIb group, positively influenced the senescence and pathogen defense-associated PR1 promoter activity, most likely involving NPR1 function (Robatzek and Somssich, 2002). In this paper, the over expression of ginkgo confirmed that WRKY genes has a certain relationship with the regulation mechanism of disease-resistant defense reaction of PR1 promoter.

\section{Conclusion}

GbWRKY31 with an ORF about $1281 \mathrm{bp}$, encoding 427 amino acids was isolated by RT-PCR from Ginkgo biloba. The amino acids of GbWRKY31 contain a conserved WRKY-motif and a Zinc-finger motif displays extensive homology to WRKY transcription factors from other plants that will enable us to conclude the diseaseresistant defense reaction of G. biloba. The phylogenetic tree analysis demonstrated the GbWRKY31 protein belongs to the WRKY IIb group. The GbWRKY31 was likely to participate in regulating thePR 1 promoter activity of disease-resistant defense reaction in ginkgo.

\section{Conflict of interest statement}

Authors declare that they have no conflict of interest.

\section{Acknowledgement}

This work was supported by National Natural Science Foundation of China (31370680) and the Natural Science Foundation of Hubei Province (No. 2013CFA039).

\section{References}

Cheng, H., Li, L., Xu, F., Cheng, S., Cao, F., Wang, Y., Wu, C., 2013. Expression patterns of a cinnamyl alcohol dehydrogenase gene involved in lignin biosynthesis and environmental stress in Ginkgo biloba. Mol. Biol. Rep. 40(1), 707-721.

Ciolkowski, I., Wanke, D., Birkenbihl, R.P., Somssich, I.E., 2008. Studies on DNA-binding selectivity of WRKY transcription factors lend structural clues into WRKYdomain function. Plant Mol. Biol. 68(1), 81-92.

Devaiah, B.N., Karthikeyan, A.S., Raghothama, K.G., 2007. WRKY75 transcription factor is a modulator of phosphate acquisition and root development in Arabidopsis. Plant Physiol. 143(4), 1789-1801. 
Eulgem, T., Rushton, P.J., Robatzek, S., Somssich, I.E., 2000. The WRKY super family of plant transcription factors. Trends Plant Sci. 5(5), 199-206.

Eulgem, T., Somssich, I.E., 2007. Networks of WRKY transcription factors in defense signaling. Curr. Opin. Plant Biol. 10(4), 366-371.

He, X., Huang, W., Chen, W., Dong, T., Liu, C., Chen, Z., Xu, S., Ruan, Y., 2009. Changes of main secondary metabolites in leaves of Ginkgo biloba in response to ozone fumigation. J. Environ. Sci. 21(2), 199-203.

Li, J., Brader, G., Kariola, T., Tapio Palva, E., 2006. WRKY70 modulates the selection of signaling pathways in plant defense. Plant J. 46(3), 477-491.

Li, S., Zhang, P., Zhang, M., Fu, C., Yu, L., 2013. Functional analysis of a WRKY transcription factor involved in transcriptional activation of the DBAT gene in Taxus chinensis. Plant Biol. 15(1), 19-26.

Liu, X., Bai, X., Wang, X., Chu, C., 2007. OsWRKY71, a rice transcription factor, is involved in rice defense response. J. Plant Physiol. 164(8), 969-979.

Maleck, K., Levine, A., Eulgem, T., Morgan, A., Schmid, J., Lawton, K.A., Dietrich, R.A., 2000. The transcriptome of Arabidopsis thaliana during systemic acquired resistance.
Nat. Genet. 26(4), 403-410.

Peng, Y., Bartley, L.E., Chen, X., Dardick, C., Chern, M., Ruan, R., Ronald, P.C., 2008. OsWRKY62 is a negative regulator of basal and Xa21-mediated defense against Xanthomonas oryzae pv. oryzae in rice. Mol. Plant. 1(3), 446-458

Ramiro, D., Jalloul, A., Petitot, A.S., De Sá, M.F.G., Maluf, M.P., Fernandez, D., 2010. Identification of coffee WRKY transcription factor genes and expression profiling in resistance responses to pathogens. Tree Genet. Genomes. 6(5), 767-781.

Robatzek, S., Somssich, I.E., 2002. Targets of AtWRKY6 regulation during plant senescence and pathogen defense. Gene. Dev. 16(9), 1139-1149.

Rushton, D.L., Tripathi, P., Rabara, R.C., Lin, J., Ringler, P., Boken, A.K., Chen, X., 2012. WRKY transcription factors: key components in abscisic acid signalling. Plant Biotechnol. J. 10(1), 2-11.

Rushton, P.J., Torres, J.T., Parniske, M., Wernert, P., Hahlbrock, K., Somssich, I.E., 1996. Interaction of elicitor-induced DNA-binding proteins with elicitor response elements in the promoters of parsley PR1 genes. EMBO J. 15(20), 5690.

\section{How to cite this article:}

Li, X., Ye, J., Wang, X., Zhang, W., Xu, F., 2016. Molecular cloning and sequence analysis of GbWRKY31, a novel transcription factor gene from Ginkgo biloba. Int. J. Curr. Res. Biosci. Plant Biol. 3(7), 90-94. doi: http://dx.doi.org/10.20546/ijcrbp.2016.307.013 